\title{
Spatially resolved femtosecond spectroscopy beyond the diffraction limit
}

\author{
U Siegner $^{1}$, M Achermann ${ }^{2}$ and U Keller ${ }^{2}$ \\ ${ }^{1}$ Physikalisch-Technische Bundesanstalt, Bundesallee 100, D-38116 Braunschweig, \\ Germany \\ ${ }^{2}$ Swiss Federal Institute of Technology Zürich, Institute of Quantum Electronics, \\ ETH Honggerberg-HPT, CH-8093 Zürich, Switzerland
}

Received 4 January 2001, accepted for publication 24 April 2001

Published 9 October 2001

Online at stacks.iop.org/MST/12/1847

\begin{abstract}
Femtosecond spectroscopy with $250 \mathrm{fs}$ temporal and $150 \mathrm{~nm}$ spatial resolution is made possible by combining near-field scanning optical microscopy with ultrafast pump-probe techniques. Femtosecond-resolved near-field microscopy allows transport studies in nanostructured materials as well as the study of carrier dynamics in single nanostructures with unprecedented temporal and spatial resolution. Measurements on single nanostructures are a powerful means for the characterization of the inhomogeneity of nanostructure ensembles. We describe the design and performance of femtosecond near-field scanning optical microscopes and give examples of typical femtosecond-resolved near-field experiments on semiconductor nanostructures.
\end{abstract}

Keywords: ultrafast spectroscopy, near-field scanning optical microscopy, semiconductor nanostructures

\section{Introduction}

Many fundamental physical processes in condensed matter and molecules proceed on femtosecond or picosecond time scales. Examples include the vibrational motion of molecules, redistribution of charge carriers in the bands of semiconductors and electron dynamics in metals. As a consequence, femtosecond laser spectroscopy has substantially contributed to our understanding of fundamental physics as well as to the characterization and optimization of electronic and optoelectronic devices [1-3]. In most femtosecond-resolved experiments, the information about the dynamic processes is spatially averaged. Spatial averaging occurs over an area of typical diameter $10-100 \mu \mathrm{m}$, determined by the diameter of the femtosecond laser beam with which the experiment is performed. This averaging is particularly disadvantageous if dynamic processes are investigated in nanostructured materials since their inherent inhomogeneity cannot be spatially resolved. Given the great potential of nanostructured devices for applications in photonics and electronics [4], the development of femtosecond-resolved measurement techniques with nanometre spatial resolution is called for. The same conclusion is reached if one considers studies of real-space transport. For example, an electron moving in the semiconductor GaAs at the saturation drift velocity of approximately $10^{7} \mathrm{~cm} \mathrm{~s}^{-1}$ moves over a distance on the order of $100 \mathrm{~nm}$ in $1 \mathrm{ps}$. Thus, time-resolved studies of such transport processes will substantially benefit from nanometrescale spatial resolution. The importance of spatial resolution in studies of electron transport has recently been highlighted for the example of optoelectronic switches [5].

Nanometre-scale spatial resolution can be provided only by techniques that beat the diffraction limit for visible or near-infrared light with which most femtosecond-resolved experiments are performed. Spatial resolution beyond the diffraction limit is routinely obtained in near-field scanning optical microscopy (NSOM) [6,7]. Moreover, other nonoptical scanning probe techniques, such as scanning tunnelling [8] and scanning force [9] microscopy, can also provide the required spatial resolution. Therefore, the combination of ultrafast spectroscopic techniques with scanning probe microscopy is a promising approach for simultaneously obtaining outstanding temporal and spatial resolution. Both time-resolved scanning force $[10,11]$ and scanning tunnelling microscopy [12-19] have been known since the early 1990s. However, since these techniques rely either on photoconductive switches [20] as time-gating devices or on electronics, it seems doubtful whether the temporal resolution 
can be pushed to the 100 fs range. All-optical techniques based on NSOM are much more promising in this respect. In fact, femtosecond-resolved NSOM experiments have been reported by several groups [21-34]. Thus, time-resolved alloptical near-field microscopy has by now been established as an experimental technique that provides both spatial resolution beyond the diffraction limit and true femtosecond temporal resolution. We would like to add that femtosecond-resolved experiments using solid immersion lenses have been pursued as an alternative approach to obtain high spatial and high temporal resolution in all-optical experiments [35].

In this paper, we describe the design and performance of the femtosecond optical near-field microscope that has recently been developed at the Swiss Federal Institute of Technology Zürich [26,27]. The design and performance issues are addressed in section 2 of this paper, where it is shown that the instrument can resolve spatiotemporal carrier dynamics with $150 \mathrm{~nm}$ spatial and $250 \mathrm{fs}$ temporal resolution.

Typical ultrafast near-field experiments can be placed into two categories: (i) studies of real-space carrier transport and (ii) measurements on single nanostructures. As discussed in section 3, we have performed transport studies with two different structures. Subsection 3.1 deals with diffusive carrier transport in semiconductor quantum wells, which have been patterned by local ion implantation [28]. In subsection 3.2, we discuss carrier drift and diffusion in metal-semiconductor composite materials which are characterized by the presence of buried Schottky contacts and built-in electrical fields [31].

Measurements on single nanostructures aim to provide a better understanding of the intrinsic properties of these structures. Often, insight into the intrinsic properties is difficult to obtain from far-field experiments, which average over the response of an ensemble of nanostructures. This averaging complicates the interpretation of the experimental data. Moreover, measurements on single nanostructures allow the characterization of the inhomogeneity of the structures. In section 4 of this paper, we present experiments on single semiconductor quantum wires [30]. The analysis of the experimental data demonstrates that femtosecond NSOM measurements allow one to quantitatively determine fluctuations of optical transition energies along a single quantum wire with nanometre-scale spatial resolution. Moreover, information on the fluctuation in thickness of the wire can be obtained from these measurements. Finally, we present our conclusions in section 5 .

\section{Experimental details}

\subsection{The design of the femtosecond near-field scanning optical microscope}

The most established method for measuring near-field optical signals [36] is NSOM [6,7], in which a nanometre-scale aperture at the end of an optical fibre is scanned in the nearfield over a sample. This NSOM technique has also been used in the femtosecond near-field measurements reported so far [21-34]. In order to obtain femtosecond temporal resolution, in all but a few cases the pump-probe technique has been combined with NSOM [21,22,24-32,34]. In the context of this paper, 'pump-probe' refers to measurements

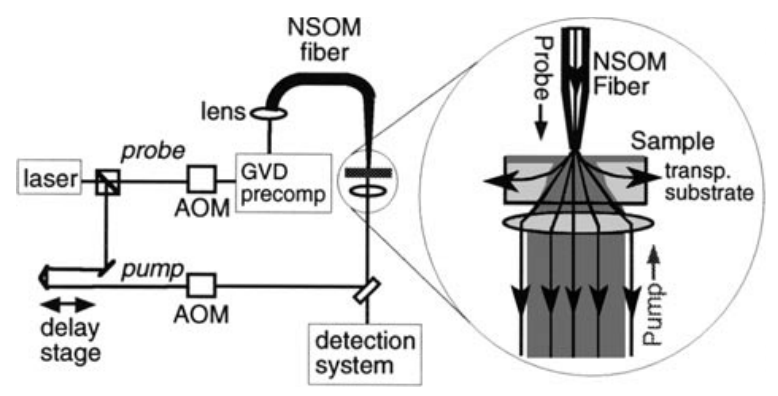

Figure 1. Right-hand side: the far-field pump/near-field transmitted probe configuration. Left-hand side: the experimental set-up. AOM, acousto-optical modulator; GVD, group velocity dispersion.

of either the differential reflectivity or the differential transmission [1,37]. In these pump-probe experiments, a pump pulse excited carriers in the sample. These excited carriers modify the absorption and the refractive index and, in turn, the reflectivity and the transmission of the sample. The pump-induced changes in reflectivity or transmission are measured by a probe pulse at various time delays $\Delta t$ after the pump excitation. More specifically, the pump-induced changes of the reflected or transmitted probe intensity are determined. They are proportional to the 'differential reflectivity' $\Delta R=R_{\text {pump on }}-R$ and the 'differential transmission' $\Delta T=T_{\text {pump on }}-T$, respectively. Here, $T$ is the linear transmission of the sample and $R$ is its linear reflectivity. The temporal resolution in these experiments is determined only by the width of the pump and probe pulses. In this paper, we will mainly concentrate on the differential transmission technique. Under a few simplifying assumptions, the differential transmission signal is a measure of the carrier density [1]. As a consequence, measurements of the differential transmission versus the time delay between the pump and probe pulses reveal the temporal evolution of the carrier distribution.

One can envisage various configurations combining femtosecond pump-probe techniques with NSOM. For example, the pump pulse, the incident probe pulse, or both pulses can propagate through the NSOM fibre while the transmitted or reflected probe pulse is detected in the far field. Alternatively, the transmitted or reflected probe pulse can be picked up by the NSOM fibre while the excitation pulses propagate in the far-field. If not mentioned otherwise, our femtosecond NSOM experiments are performed in the far-field pump/near-field transmitted probe configuration shown on the right-hand side of figure 1 . The sample is excited from the bottom side by a pump pulse that is focused by far-field optics. The pump-induced changes in transmission are detected by a near-field probe pulse that is sent through the NSOM fibre and transmitted through the sample. All experiments are performed at room temperature.

The NSOM used in our experiments is a home-built instrument with aluminium coated pulled [7] or tube-etched $[38,39]$ NSOM tips. The aperture of the tips is held within $\simeq 10 \mathrm{~nm}$ of the surface of the sample using shear-force feedback [40]. The shear force is detected by a piezoelectric tuning fork [41] vibrated at around $33 \mathrm{kHz}$ in order to avoid the effects of scattered light which can occur in optical feedback techniques. Both the feedback-controlled tip-sample flying height and the 
scanning of the sample are controlled by commercial Park Scientific Instruments scanning force microscope electronics, which was adapted for the NSOM.

Details of the pump-probe NSOM set-up are shown on the left-hand side of figure 1. A modelocked laser source (either a home-built Ti:sapphire or a commercial Cr:LiSAF laser) generates a train of $\simeq 100 \mathrm{fs}$ pulses at around $800 \mathrm{~nm}$ at a repetition rate of $\simeq 100 \mathrm{MHz}$. The pulse train is split into pump and probe beams by a polarizing beamsplitter cube. The pump beam is sent through a motorized variable delay stage, which determines the time delay between the pump and probe pulses. It is then square-wave modulated at $1.00 \mathrm{MHz}$ by an acoustooptic modulator (AOM) from IntraAction Corp (model AOM$40 \mathrm{~N}$ ) before being sent up through a $40 \times$ microscope objective that focuses the beam to $\mathrm{a} \simeq 10 \mu \mathrm{m}$ spot on the sample. The probe pulse is first square-wave modulated at $1.05 \mathrm{MHz}$ by an $\mathrm{AOM}$ and then sent through a group velocity dispersion (GVD) precompensation set-up. This set-up consists of two prisms, which introduce a negative frequency chirp [42]. The chirped probe pulse is then coupled into a single-mode NSOM fibre, emerging at the output NSOM tip aperture with pulse widths typically below $\simeq 200$ fs [27] since the negative chirp of the pulse and the positive GVD of the fibre are well balanced. The output from the NSOM tip aperture is transmitted through the thin sample in the near field and collected in the far field through the microscope objective. The probe pulses are then sent to the detection optics and an avalanche photodiode (a low-noise home-built module) that serves as a photodetector. Finally, the differential transmission signal is measured at the pump-probe difference frequency of $50 \mathrm{kHz}$ with a lock-in amplifier (Stanford Research Systems SR830).

\subsection{The performance of the femtosecond near-field scanning optical microscope}

A very important issue in femtosecond NSOM experiments is the signal-to-noise ratio. In order to improve the signalto-noise ratio, lock-in detection is used as in most farfield pump-probe measurements. Moreover, great care has been taken to reduce the amount of pump light that is backscattered into the detector where it contributes to the shot noise and the laser noise floor. A large reduction of the amount of backscattered pump light is achieved by polarization discrimination and spatial filtering in a confocal set-up [27]. With these precautions, differential transmission signals as small as $\Delta T / T \simeq 10^{-4}$ can routinely be measured. A detailed discussion of the noise suppression techniques and other noise considerations can be found in [27].

In order to prove the imaging capability of the femtosecond NSOM, experiments on semiconductor nanostructures have been performed. These structures have been obtained by nanometre-scale lateral patterning of an undoped $80 \AA \mathrm{GaAs} / \mathrm{Al}_{0.3} \mathrm{Ga}_{0.7}$ As single quantum well with focused ion beam (FIB) implantation of $50 \mathrm{keV}$ Ga ions. The implantation energy was chosen in order to create damage in the GaAs quantum well, according to TRIM simulations [43]. The local ion implantation results in the formation of defects, which gives rise to carrier trapping with picosecond or sub-picosecond time constants $[44,45]$. Sample $100 / 2000$ is patterned with $100 \mathrm{~nm}$ implanted stripes and $2000 \mathrm{~nm}$ spaces at a dose of

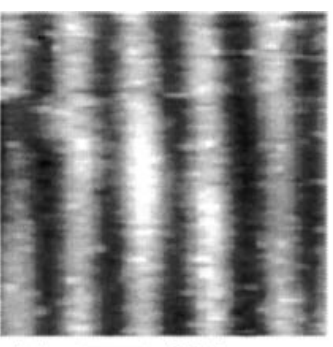

(a)

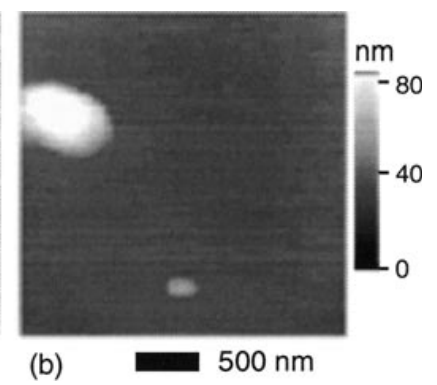

(b)

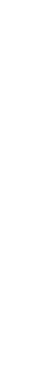

Figure 2. (a) A two-dimensional image of the pump-probe amplitude at zero time delay. (b) The simultaneously measured topography of the GaAs/AlGaAs quantum well with $200 \mathrm{~nm}$ FIB implanted stripes and $400 \mathrm{~nm}$ spaces.

$8 \times 10^{11} \mathrm{~cm}^{-2}$, sample 200/400 with $200 \mathrm{~nm}$ implanted stripes and $400 \mathrm{~nm}$ spaces at a dose of $3 \times 10^{12} \mathrm{~cm}^{-2}$. The FIB implantation creates a spatial defect profile with well-defined edges since TRIM simulations [43] show that the defects spread only $20 \mathrm{~nm}$ beyond the nominal width of the implanted stripes. To prepare the samples for transmission experiments, each quantum well sample was glued onto a glass disc with the epitaxial layers facing downwards. Subsequently the GaAs substrate and $\mathrm{AlGaAs} / \mathrm{GaAs}$ etch-stop layers were removed by chemical wet etching with $\mathrm{H}_{2} \mathrm{O}_{2} / \mathrm{NH}_{4} \mathrm{OH}$ [46] solution for GaAs and $\mathrm{HCl}$ solution for AlGaAs. The remaining quantum well has a $12 \mathrm{~nm}$ AlGaAs top barrier, which allows one to bring the NSOM tip close to the optically active layer, ensuring that high spatial resolution is obtained in near-field experiments. In all experiments, the laser was tuned to the lowest heavy hole exciton resonance of the quantum well at $1.475 \mathrm{eV}(840 \mathrm{~nm})$. The pump fluence was $4 \mu \mathrm{J} \mathrm{cm}^{-2}$, corresponding to a carrier density of about $10^{11} \mathrm{~cm}^{-2}$.

Figure 2(a) shows a two-dimensional image of the amplitude of the pump-probe signal at zero time delay, measured in the configuration of figure 1 on sample 200/400. One can clearly distinguish variations in the pump-probe amplitude image which follow the $600 \mathrm{~nm}$ period of the FIB implantation pattern, where the dark regions correspond to smaller signals. Fast carrier trapping in the implanted stripes is at the origin of the pump-probe pattern. Carrier trapping reduces the carrier density in the implanted stripes even at zero time delay when the pump and probe pulses temporally overlap, resulting in a smaller pump-probe amplitude. It should be noted that no contrast is seen in the simultaneously measured topography image of figure $2(b)$, excluding the possibility that the pattern in the pump-probe image is a topographical artefact [47]. Thus, femtosecond NSOM can provide images of the optical nonlinearity at fixed time delays.

The spatial resolution of these images can be determined if the amplitude of the pump-probe signal is plotted versus position. Such a plot is shown in the inset of figure 3 for the FIB implanted quantum well sample 200/400. Taking the 10-90\% rise of the amplitude as the measure of the spatial resolution, a resolution of $\simeq 150 \mathrm{~nm}$ is obtained. The temporal resolution in femtosecond NSOM experiments can be determined from the $10-90 \%$ rise time of pump-probe traces. Figure 3 shows the pump-probe signal versus time delay, measured at a fixed position on the FIB implanted quantum well sample 100/2000. From this trace, a temporal resolution of $\simeq 250 \mathrm{fs}$ is determined. 


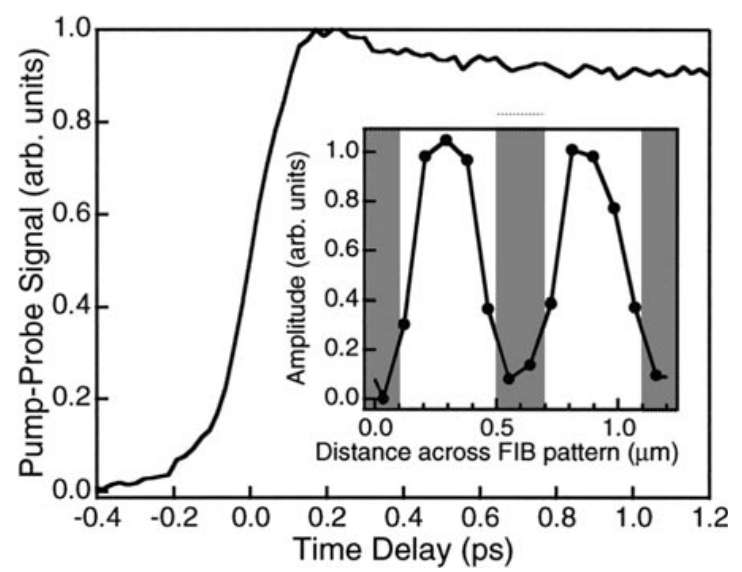

Figure 3. A near-field pump-probe trace of the FIB implanted quantum well sample 100/2000. The inset shows the pump-probe amplitude at zero time delay versus distance across the FIB pattern for the quantum well sample 200/400. The grey regions correspond to the implanted stripes.

The temporal resolution in the pump-probe measurements is slightly worse than the width of the probe pulse due to the broadening of the pump pulse by GVD in the AOM. Thus, the temporal resolution could be improved if the GVD of the AOM were compensated in the pump beam. For the experiments discussed in this paper, no improvement of the temporal resolution is necessary.

Summarizing this section, the femtosecond NSOM allows differential transmission measurements with $150 \mathrm{~nm}$ spatial and $250 \mathrm{fs}$ temporal resolution and a high sensitivity of $\Delta T / T \simeq 10^{-4}$.

\section{Transport studies}

\subsection{Diffusion in locally patterned semiconductor quantum wells}

The FIB implanted quantum wells provide a well-suited arena in which to demonstrate the potential of femtosecond NSOM for studies of carrier transport. In the following, we will discuss the fact that the high spatial and temporal resolution of femtosecond NSOM allows one to obtain insight into the details of carrier diffusion in locally patterned semiconductors. The carrier diffusion studies have been performed on the FIB implanted quantum well samples described in the previous section.

Figure 4 shows pump-probe signals (full lines) from sample 100/2000 versus distance across the FIB implanted stripes for fixed time delays $\Delta t$. After a small time delay, $\Delta t=4 \mathrm{ps}$, we observe a square-like pump-probe profile, corresponding to a square-like carrier density profile. The minimum of the profile is located at the position of the implanted stripe where fast trapping of carriers by defects has substantially reduced the carrier density. After a greater time delay, $\Delta t=80 \mathrm{ps}$, a smoother, quasi-sinusoidal profile is observed. The reason for the temporal evolution of the carrier density profile is that strong carrier diffusion occurs at the edges of the initial square-like profile [28]. Diffusion is driven by the steep gradient of the carrier density close to the implanted stripes. The detailed shape of the carrier density profile and its

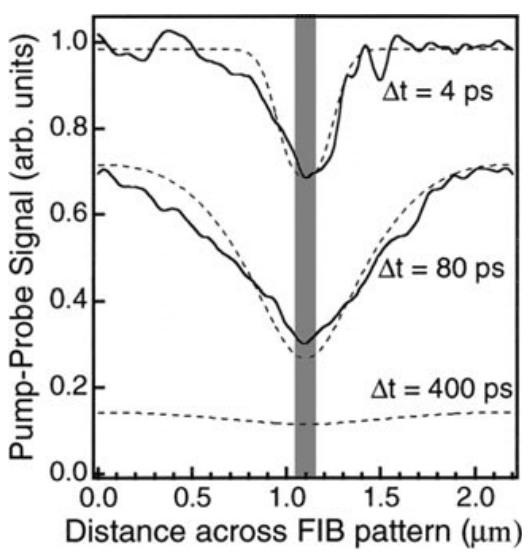

Figure 4. The measured (full lines) and calculated (broken lines) pump-probe signals versus position for various time delays for a FIB patterned quantum well sample with $100 \mathrm{~nm}$ implanted stripes and $2000 \mathrm{~nm}$ spaces. The grey region corresponds to an implanted stripe. Averaging over a $300 \mathrm{~nm}$ tip aperture was assumed in the calculation.

temporal evolution could not have been observed in far-field pump-probe experiments.

A detailed analysis of the near-field data can be performed with the continuity equation. Carrier diffusion, trapping in the implanted stripes and recombination are included in the analysis. Carrier drift can be neglected because the FIB implanted quantum well sample does not contain large potential gradients [28]. Since the optical excitation of excitons is followed by ionization after about $300 \mathrm{fs}$ at room temperature [48], the dynamics of the density $n$ of band electron-hole pairs is modelled. We have solved the onedimensional continuity equation for the electron-hole pair density $n$, coupled to the differential equation for the density $n_{t}$ of occupied traps

$$
\begin{gathered}
\frac{\partial n(x, t)}{\partial t}=D_{a} \frac{\partial^{2} n}{\partial x^{2}}-\frac{n}{\tau_{r e c}}-\frac{n}{\tau_{t}(x)}\left(1-\frac{n_{t}}{N_{0}}\right)+G(t) \\
\frac{\partial n_{t}(x, t)}{\partial t}=\frac{n}{\tau_{t}(x)}\left(1-\frac{n_{t}}{N_{0}}\right) .
\end{gathered}
$$

Here, $D_{a}$ is the ambipolar diffusion constant $[49,50], \tau_{\text {rec }}$ the recombination time, $\tau_{t}$ the trapping time and $N_{0}$ the total density of traps in the implanted stripes. The spatially uniform carrier excitation is described by the $x$ independent generation term $G(t)$, for which we assume a sech ${ }^{2}$ pulse with a full width at half maximum of $200 \mathrm{fs}$. The trapping rate $1 / \tau_{t}$ decreases from a constant value in the implanted stripes to zero outside the stripes. This decrease occurs over a $20 \mathrm{~nm}$ wide transition region, determined from the TRIM simulations. Trap filling and the corresponding decrease of the rate of trapping with time are accounted for in the third term on the right-hand side of equation (1) in conjunction with equation (2). The degree of trap filling in the implanted stripes is adjusted by the ratio $N_{0} / \int G(t) \mathrm{d} t$.

The initial exciton ionization [48] as well as the thermalization of carriers with the lattice are not included in the model. At room temperature, the latter occurs within about a picosecond if carriers are injected at the band edge of GaAs/AlGaAs quantum wells [51,52]. Therefore, 


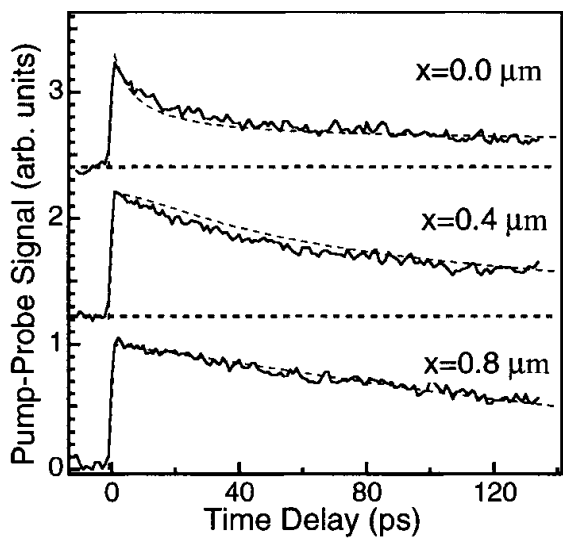

Figure 5. The measured (full lines) and calculated (broken lines) pump-probe traces for various positions for a FIB patterned quantum well sample with $100 \mathrm{~nm}$ implanted stripes and $2000 \mathrm{~nm}$ spaces. The FIB implanted stripe is located at $x=0.0 \mu \mathrm{m}$.

equations (1) and (2) are used only for time delays of $2 \mathrm{ps}$ and longer. Moreover, for these time delays, the ambipolar diffusion constant $D_{a}$ for room temperature can be used, neglecting any temperature dependence of diffusion [50].

In order to compare the calculated electron-hole pair density $n$ with the pump-probe profiles, we assume that the pump-probe signal is proportional to $n$ [1]. The calculated electron-hole pair density profiles are shown in figure 4 as broken lines. The calculation was performed with $D_{a}=10 \mathrm{~cm}^{2} \mathrm{~s}^{-1}, \tau_{\text {rec }}=250 \mathrm{ps}^{3}, \tau_{t}=3 \mathrm{ps}$ and $N_{0} / \int G(t) \mathrm{d} t=4$ to account for trap filling. The value for $D_{a}$ is reasonable in view of earlier work on GaAs/AlGaAs heterostructures and quantum wells [49,50]. The good agreement between the experimental and calculated profiles confirms that diffusion substantially affects the dynamics. The calculated carrier profile at $\Delta t=400 \mathrm{ps}$ is also shown ${ }^{4}$. This profile is almost flat since diffusion has evened out the carrier density gradients.

The complex diffusion dynamics seen in figure 4 is reflected in measurements of the pump-probe signal versus time delay at various positions. The experimental traces are shown as full curves in figure 5 . As the probe position is moved closer to the implanted stripe, a rapidly decaying contribution gains strength in the pump-probe traces. The broken curves in figure 5 have been calculated with the continuity equation and the same parameters as were used to calculate the carrier density profiles of figure 4 . Good agreement between the measured and calculated traces is obtained. If the pumpprobe traces are fitted to a double exponential function, the time constant of the fast contribution can be quantitatively determined. This time constant is considerably smaller closer to the implanted stripes, i.e. it strongly depends on position [28]. This behaviour reflects the strong diffusion at the edges of the initial square-like carrier profile.

The complex diffusion dynamics observed in figures 4 and 5 has to be compared with the well-known case of diffusion

\footnotetext{
3 The short recombination time is not due to intrinsic radiative recombination but results from nonradiative decay due to the application of a small laterally uniform background implantation dose during FIB patterning.

4 For very long time delays, the measured pump-probe signal is so small that noise prevents detailed analysis. Therefore, only the calculated profile is shown in figure 4 .
}

in a sinusoidal carrier profile [53]. In sinusoidal profiles, the time constant of the diffusion-induced decay of the carrier density does not depend on position. This case has also been observed in femtosecond NSOM experiments [28]. Thus, the high spatial and temporal resolution of femtosecond NSOM allows one to distinguish among different diffusion regimes in nanometre-scale patterned semiconductors. These results demonstrate that femtosecond NSOM is a powerful tool for studies of carrier transport.

With respect to the transport studies presented in the next subsection, we would like to emphasize that the signature of diffusive transport in square-like carrier density profiles is the spatial dependence of the decay of the pump-probe traces. The decay becomes faster as the probe position is moved towards the edge of the square-like carrier density profile.

\subsection{Drift and diffusion in metal-semiconductor composite materials}

Femtosecond NSOM can even disentangle more complex transport dynamics that involve both drift and diffusion of carriers. As an example, we will discuss transport in metalsemiconductor composite materials. This class of materials includes annealed low-temperature grown GaAs that contains metallic As precipitates [54] and lithographically fabricated nano-scale metal discs embedded in GaAs [55]. In these two materials, buried Schottky contacts and built-in electrical fields are formed at the semiconductor-metal interfaces $[54,56]$. We have performed femtosecond NSOM experiments on nanoscale metal discs embedded in GaAs. These measurements reveal the dynamics of carrier transfer from a semiconductor into embedded metal clusters in the presence of Schottky contacts and built-in electrical fields. The carrier transfer involves transport towards and trapping into the metal discs. Our experiments show that efficient transport of electrons towards the metal occurs only at relatively high carrier densities. For these densities, the built-in field is screened, allowing efficient transport of electrons. Only, in this case, the metal clusters act as efficient trapping centres for electrons. We would like to add that carrier trapping is an important issue in many applications of metal-semiconductor composite materials in electronics and optoelectronics $[57,58]$.

Our sample consists of tungsten (W) discs of $\simeq 80 \mathrm{~nm}$ diameter and thickness $20 \mathrm{~nm}$ embedded in GaAs in a rectangular pattern. The spacing between the discs is $1 \mu \mathrm{m}$. The GaAs is $\mathrm{n}$-doped (electron density $\simeq 10^{16} \mathrm{~cm}^{-3}$ ) and Schottky contacts are formed at the W/GaAs interface [56]. Opaque layers of the sample have been removed by wet etching to allow femtosecond NSOM experiments in the transmission geometry of figure 1 . More details about the growth and the structure of the sample can be found in [31]. In all NSOM pump-probe experiments the excitation photon energy is $1.46 \mathrm{eV}$, corresponding to excitation $40 \mathrm{meV}$ above the bandgap of GaAs.

Figure 6(a) shows a two-dimensional near-field image of the pump-probe signal taken after a time delay of $15 \mathrm{ps}$ with an optically excited carrier density of $\simeq 9 \times 10^{16} \mathrm{~cm}^{-3}$. The disc pattern is clearly visible. No pattern is seen in the simultaneously measured topographic image (data not shown). Moreover, the linear transmission hardly shows any contrast, 


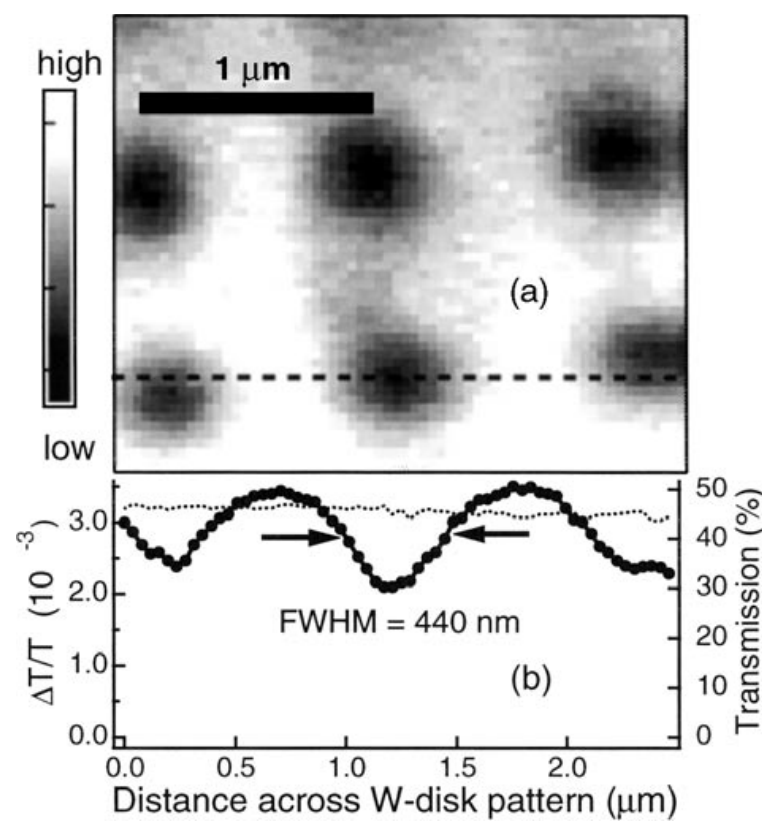

Figure 6. Results for a tungsten disc sample. (a) The two-dimensional optical near-field image of the pump-probe signal after a time delay of $\Delta t=15 \mathrm{ps}$ with a carrier density of $\simeq 9 \times 10^{16} \mathrm{~cm}^{-3}$. (b) A line scan of the pump-probe signal (filled circles) and the linear transmission (dotted line) along the dashed line in $(a)$. FWHM; full width at half maximum.

as can be seen from the line scan in figure $6(b)$. Therefore, the contrast of figure $6(a)$ is due to the variation of the optical nonlinearity, which results from the variation of the carrier density. The line scan of the pump-probe signal in figure $6(b)$ shows that the signal is reduced in a region around a $\mathrm{W}$ disc with a full width at half maximum of $440 \mathrm{~nm}$. Given the spatial resolution of $250 \mathrm{~nm}$ in this experiment, we conclude that the carrier density is reduced in a region of diameter $\simeq 360 \mathrm{~nm}$ around the discs (here Gaussian functions have been assumed). Such a carrier density profile is characteristic of diffusive transport towards a trapping centre, as can be seen from the comparison with the carrier density profiles in figure 4 .

In the following, we will show that the carrier transport substantially depends on the optically excited carrier density due to the presence of the built-in field. Figure 7 shows pumpprobe traces taken either over the discs or midway between the discs at various carrier densities. The pump-probe traces are almost constant and hardly depend on the position at lower carrier densities comparable to the background doping concentration.

We note that electrons yield the major contribution to the pump-probe traces for detection close to the band edge [59]. With respect to electron transport, one has to consider that the built-in electrical field of the Schottky contacts points towards the metal before optical excitation. In the low-density regime, the built-in field persists and drift of electrons away from the discs counteracts diffusion towards the discs. From figure 7 we conclude that this effect strongly suppresses the net electron transport at low densities. This conclusion is based on two arguments. (i) The almost constant pump-probe traces indicate that only a small fraction of the electrons is removed by rapid trapping at the $\mathrm{W} / \mathrm{GaAs}$ interface. This is because electrons cannot move towards the interface. (ii) Owing to

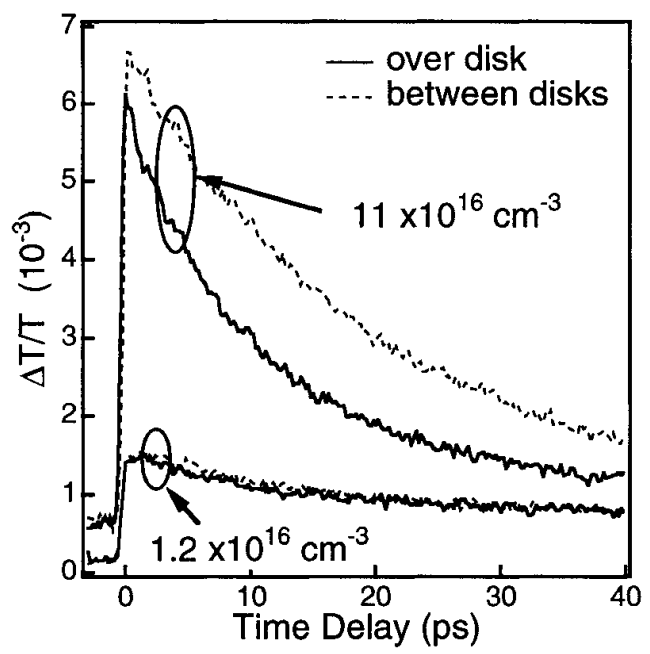

Figure 7. Near-field pump-probe traces for a tungsten disc sample taken over (full lines) and midway between (broken lines) the discs for various densities of optically excited carriers. The disc spacing is $1 \mu \mathrm{m}$.

the suppression of electron transport, no significant difference between traces at different positions is observed at low densities.

At higher densities well above the doping concentration, the pump-probe traces exhibit a pronounced fast decay superimposed on a constant offset. The time constant of the fast decay is found to decrease from 20 ps for between the discs to $11 \mathrm{ps}$ for over the discs [31]. As shown in the previous subsection, this behaviour is characteristic of diffusive transport in square-like carrier profiles. We would like to emphasize that the image of figure 6 has also been taken at higher carrier densities.

The dynamics seen at higher carrier densities is strongly affected by the motion of positively charged holes. The holes drift and diffuse towards the $\mathrm{W}$ discs where they can be trapped. Since the optically excited carrier density exceeds the doping concentration, the positive charge accumulated in the discs can substantially screen the built-in field. As a consequence, the electron drift current is reduced and efficient net electron transport takes place due to diffusion. The electrons can then be efficiently trapped into the $\mathrm{W}$ discs and recombine with the holes. At later times, the built-in field is restored since the optically excited carrier density has decreased. Then, electron transport is again suppressed, leading to the offset of the traces in figure 7.

Summarizing this subsection, femtosecond NSOM can provide detailed insight into spatiotemporal carrier dynamics in nano-scale metal-semiconductor composite systems.

\section{Studies of single semiconductor quantum wires}

Studies of single nanostructures are one of the most important applications of the femtosecond NSOM technique. Such studies allow one to gain insight into the intrinsic properties of nanostrucutres, unperturbed by spatial averaging over an ensemble of structures. Moreover, studies of single nanostructures can characterize growth inhomogeneities in nanostructured materials. In this section, we discuss 
experiments on single semiconductor quantum wires (QWRs). The QWRs have been produced by epitaxial growth on Vgroove substrates [60]. Quantum wires produced by this method are promising one-dimensional systems due to their large confinement energy [61]. We will first demonstrate that femtosecond NSOM measurements on single V-groove QWRs with the instrument described in section 2 are possible. Moreover, we present a novel method for the mapping of fluctuations in quantization energy along single quantum wires with nanometre-scale spatial resolution. This method takes advantage of the sensitive photon energy dependence of the near-field pump-probe traces around the lowest exciton resonance of the QWRs.

The sample consists of a $0.5 \mu \mathrm{m}$ pitch lateral array of single V-groove $\mathrm{GaAs} / \mathrm{Al}_{x} \mathrm{Ga}_{1-x} \mathrm{As}(x=0.33) \mathrm{QWRs}$, grown by low-pressure organometallic chemical vapour deposition (OMCVD) on a GaAs substrate [60]. Figure 8(a) shows a schematic picture of the sample before any further processing. One can seen the GaAs substrate, the AlGaAs energy barriers and, between the barriers, the GaAs quantum well with the QWR at the bottom of the V-groove. The nominal thickness of the GaAs epilayer is $1.7 \mathrm{~nm}$, resulting in a QWR of thickness $4.2 \mathrm{~nm}$. To prepare the sample for the NSOM measurements, it was mounted upside down on a glass disc and the GaAs substrate was removed by selective wet etching. The etching leaves a $35 \mathrm{~nm}$ thick $\mathrm{Al}_{x} \mathrm{Ga}_{1-x}$ As barrier layer on top of the QWRs, as shown in figure $8(b)$. The thickness of this layer was chosen to ensure both QWR energy confinement and good spatial resolution in NSOM measurements. The high quality of the etching becomes obvious from figure $8(c)$, in which the topography of the sample measured with an atomic force microscope after etching is shown. At $20 \mathrm{~K}$, the sample was characterized by photoluminescence (PL) measurements, showing the QWR peak at $1.675 \mathrm{eV}$ with a full width at half maximum (FWHM) of $14 \mathrm{meV}$. From these PL measurements and PL excitation spectra, we estimate that the lowest exciton has a transition energy of $1.59-1.60 \mathrm{eV}$ at room temperature.

The femtosecond NSOM pump-probe experiments on the QWRs have been performed with 200 fs pulses, which have a spectral FWHM of $10 \mathrm{meV}$. In a first experiment, the pump pulse with centre photon energy $1.58 \mathrm{eV}$ is transmitted through the NSOM tip to excite the QWRs locally. The excited carrier density was calculated to be about $10^{5} \mathrm{~cm}^{-1}$. The pumpinduced changes of the reflected probe pulse intensity are globally measured in the far field. This configuration for the measurement of the differential reflectivity is different from the femtosecond NSOM configuration used so far, emphasizing that the femtosecond NSOM technique can be successfully implemented in various configurations. Figure 9 shows a twodimensional image of the differential reflectivity signal with zero time delay. The quantum wires, separated by a distance of $0.5 \mu \mathrm{m}$, are well discernable, proving that high spatial resolution is achieved. Along a single wire, intensity variations of the pump-probe signal indicate fluctuations of the energy or the oscillator strength of the QWR excitonic resonance.

The inset of figure 9 shows a line scan perpendicular to the QWRs. Between the QWRs the pump-probe signal completely disappears, proving that high spatial resolution and strong contrast are obtained in the pump-probe experiment. In contrast, the image of the transmitted pump intensity shows a
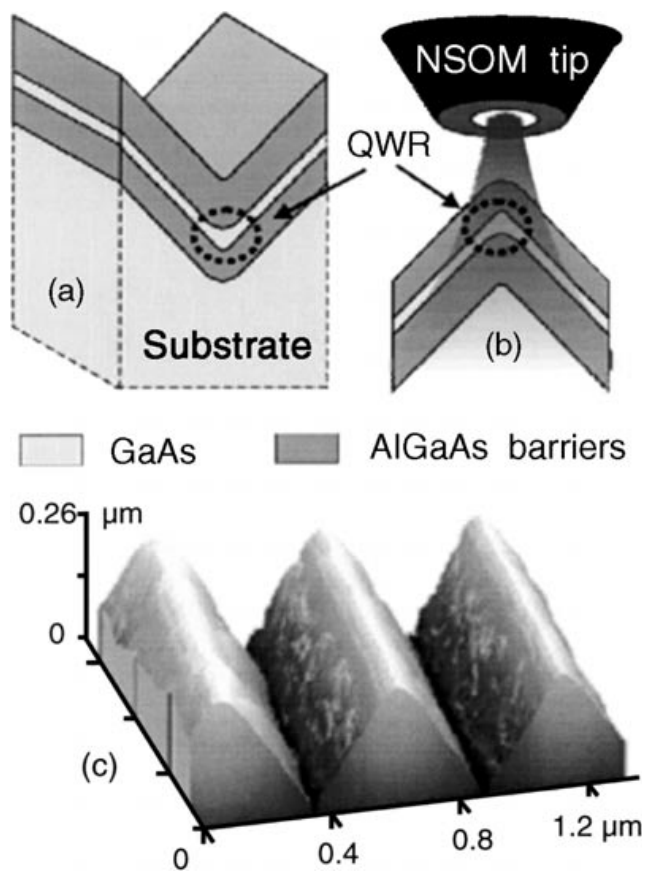

Figure 8. V-groove quantum wires. Schematic diagrams of the QWR sample (a) before and $(b)$ after etching with its orientation regarding the NSOM tip. (c) A two-dimensional AFM image of the topography of the QWR sample after etching.

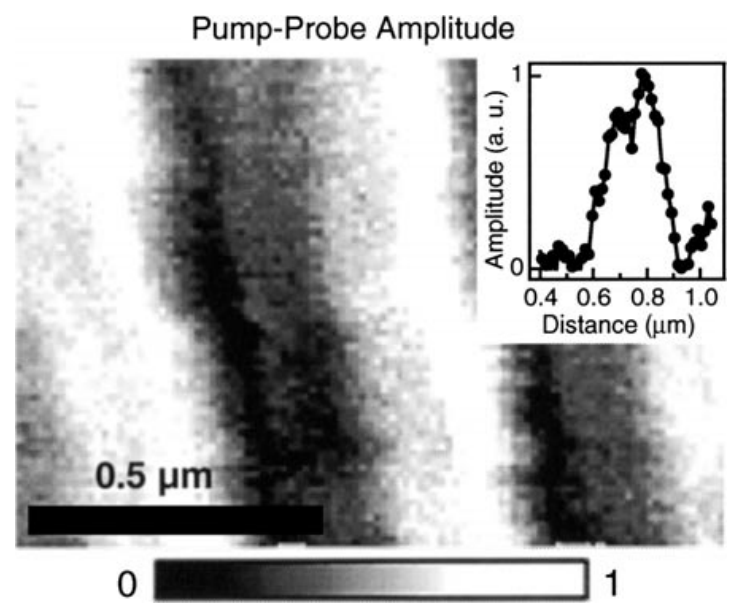

Figure 9. V-groove quantum wires. A two-dimensional image of the pump-probe amplitude at zero time delay. The bending of the wires in the centre of the image is due to scanner drift. The inset shows a line scan of the pump-probe amplitude perpendicular to a single quantum wire.

modulation of only a few per cent. Therefore, the coupling of the near-field pump and the sample changes only slightly across the sample and topographical artefacts [47] yield a negligible contribution to the nonlinear probe modulation seen in figure 9. Moreover, in scans along single QWRs, the topographical artefacts will be even more strongly reduced compared with those in scans perpendicular to the QWRs. Thus, nonlinear optical near-field pump-probe measurements lend themselves well to the characterization of QWRs.

The fluctuations in quantization energy along a single QWR are determined from measurements in the standard femtosecond NSOM configuration of figure 1 . The aperture of 

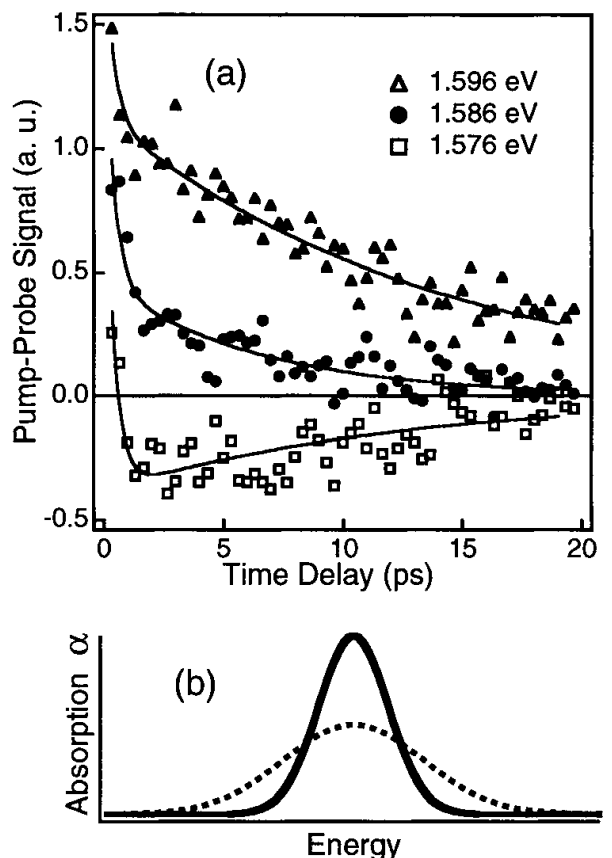

Figure 10. V-groove quantum wire. (a) Near-field pump-probe traces taken at the same position of the QWR but at various photon energies around the lowest exciton resonance. (b) A schematic picture showing an absorption resonance $\alpha$ before pump excitation (full curve) and the broadened resonance after pump excitation (broken curve).

the NSOM tip is $\simeq 200 \mathrm{~nm}$ in these experiments. For resonant excitation of a QWR, the differential transmission is mainly due to pump-induced changes in absorption and its decay reflects the carrier dynamics in the QWR [29]. Figure 10(a) shows near-field pump-probe traces for various laser photon energies around the lowest exciton resonance. The traces are taken at a fixed position on a single wire. The measurements are performed with constant laser excitation power, resulting in a carrier density of $2 \times 10^{6} \mathrm{~cm}^{-1}$ for resonant excitation of the exciton. For all photon energies, a positive bleaching signal is observed at early times due to excitonic phase space filling and screening. The bleaching signals exhibit a fast initial decay with a time constant of 300-400 fs. We attribute this decay to ionization of excitons by LO phonons, which reduces the phase space filling contribution to the pump-probe signal [48].

After the initial fast decay, the pump-probe traces sensitively depend on the laser photon energy. The nonlinear signal is positive at higher photon energies close to the exciton resonance. This behaviour indicates a bleaching process, i.e. a decrease of the absorption. In contrast, at photon energies well below the exciton resonance, the signal becomes negative, demonstrating that induced absorption is occurring. We attribute the induced absorption to pump-induced broadening of the exciton resonance. Figure 10(b) schematically shows the broadening of an absorption resonance due to excited carriers, resulting in an increase of the absorption on the lowenergy side. For all photon energies, the pump-probe signal approaches zero with a second time constant of 10-12 ps. Since radiative recombination occurs on a much longer time scale [62], the slow decay is due to nonradiative processes,

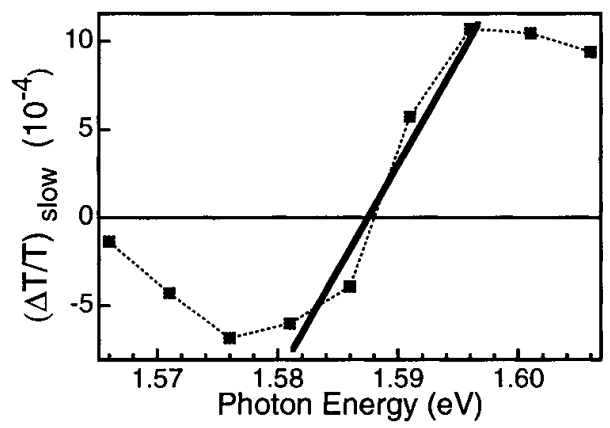

Figure 11. V-groove quantum wire. The amplitude $(\Delta T / T)_{\text {slow }}$ of the slowly decaying contribution of near-field pump-probe traces versus the photon energy for a fixed QWR position. The full line indicates the slope used to calculate the variations in quantization energy.

such as fast carrier trapping by activated interface impurities [63].

In order to quantify the dependence on detuning, we have fitted the pump-probe traces to double exponential functions $(\Delta T / T)_{\text {fast }} \exp \left(-\Delta t / \tau_{\text {fast }}\right)+(\Delta T / T)_{\text {slow }} \exp \left(-\Delta t / \tau_{\text {slow }}\right)$. In figure 11, the amplitude $(\Delta T / T)_{\text {slow }}$ of the slowly decaying contribution is shown versus the laser photon energy. These data have been obtained from the analysis of a large set of pump-probe traces measured at a fixed position on the QWR, i.e. for a fixed exciton resonance energy ${ }^{5}$. In a photon energy region around the zero crossing of the amplitude $(\Delta T / T)_{\text {slow }}$ the shape of the pump-probe signal is very sensitive to the photon energy. In this region we obtain, to a first approximation, a linear relation between the amplitude and the energy detuning $\left(\hbar \omega-E_{x}\right)$ with the following slope (the full line in figure 11):

$$
\Delta\left[(\Delta T / T)_{\text {slow }}\right] / \Delta\left(\hbar \omega-E_{x}\right)=1.2 \times 10^{-4} \mathrm{meV}^{-1} .
$$

Here, $\hbar \omega$ is the laser photon energy and $E_{x}$ is the resonance energy of the exciton. From equation (3), one can calculate the variation of the energy detuning for a given change of the amplitude. Equation (3) is valid for a certain excitation power. If this excitation power is used, equation (3) allows the mapping of fluctuations in quantization energy along a single quantum wire. This procedure will be discussed in the following.

If the NSOM tip is scanned along a QWR for a fixed laser photon energy, the detuning between the photon energy $\hbar \omega$ and the exciton resonance energy $E_{x}$ may change due to fluctuations of the exciton quantization energy. In turn, the change of the detuning will substantially change the shape of the pump-probe traces. This effect is illustrated in figures 12(a) and $(b)$, which show pump-probe traces taken at two different positions for a fixed laser photon energy $\hbar \omega=1.578 \mathrm{eV}$. For the quantitative determination of the exciton energy fluctuations, the change of the amplitude $(\Delta T / T)_{\text {slow }}$ is considered. Figure 12(c) shows $(\Delta T / T)_{\text {slow }}$ versus position along a single $Q W R$ for the fixed photon energy $1.578 \mathrm{eV}$. The values of $(\Delta T / T)_{\text {slow }}$ are calculated from pump-probe traces taken every $200 \mathrm{~nm}$ along a single QWR

5 The data of figures $10(a)$ and 11 have been taken at different positions. This explains why the amplitude at $1.586 \mathrm{eV}$ is positive in figure $10(a)$ but negative in figure 11 . 

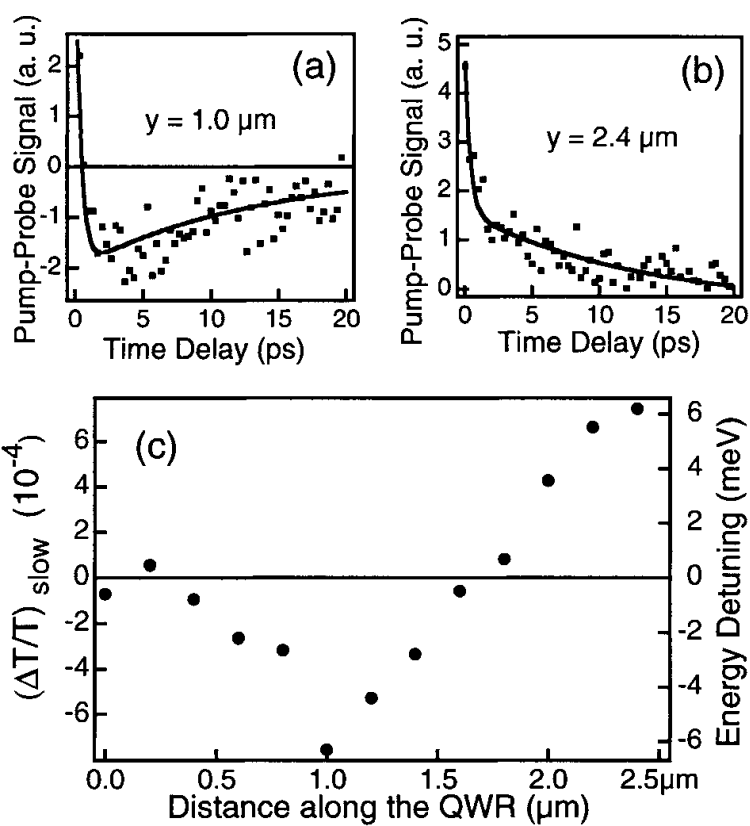

Figure 12. V-groove quantum wire. Pump-probe traces $(a)$ and $(b)$ for two different positions $y$ of a single quantum wire for a fixed laser photon energy of $1.578 \mathrm{eV}$. (c) The amplitude $(\Delta T / T)_{\text {slow }}$ of the slowly decaying contribution of near-field pump-probe traces versus position along a single QWR at the fixed laser photon energy of $1.578 \mathrm{eV}$. The right-hand vertical axis shows the calculated energy detuning variation along the wire.

with a NSOM tip providing a spatial resolution of $\simeq 200 \mathrm{~nm}$. As expected, $(\Delta T / T)_{\text {slow }}$ varies with position. We would like to emphasize that the variation of $(\Delta T / T)_{\text {slow }}$ in figure $12(c)$ is due to variations in detuning that result from the fluctuation of the exciton resonance energy since the photon energy is constant.

The fixed photon energy of $1.578 \mathrm{eV}$ is chosen to given the smallest amplitude $(\Delta T / T)_{\text {slow }}$ at the starting position of the scan. This ensures that equation (3) is valid. Using equation (3), we can calculate the change of the detuning between the photon energy and the exciton resonance energy from the variation of $(\Delta T / T)_{\text {slow }}$. Following this procedure, the right-hand vertical axis of figure $12(c)$ has been obtained. From this axis, it can be determined how the energy detuning changes with position along a single quantum wire. The change of the energy detuning is equal to the change of the exciton quantization energy. We note that an increase (decrease) of $(\Delta T / T)_{\text {slow }}$ reflects a red shift (blue shift) of the excitonic resonance and, therefore, a smaller (larger) quantization energy.

Energy variations of $12 \mathrm{meV}$ are observed over a wire length of $2 \mu \mathrm{m}$. Such energy variations are often deduced from the inhomogeneous broadening of spatially integrated PL spectra. At low temperatures, the FWHM of the QWR PL peak is $14 \mathrm{meV}$, directly giving the inhomogeneous broadening. From the comparison with the measured fluctuations in quantization energy of $12 \mathrm{meV}$, we conclude that the inhomogeneous broadening mainly arises from energy fluctuations along the wires. The fluctuations in quantization energy result from variations in thickness of the wire. Considering the first electron-heavy hole transition in a simple finite potential model for the V-groove QWRs, the energy variation of $12 \mathrm{meV}$ corresponds to a fluctuation in wire thickness of about $3 \AA$ or one monolayer of GaAs. It is noteworthy that energy fluctuations of less than $12 \mathrm{meV}$ are resolved in figure 12(c). These fluctuations correspond to variations in thickness of less than one monolayer, showing that monolayer fluctuations are averaged over the diameter of the NSOM tip. Therefore, the monolayer fluctuations have to occur on a length scale smaller than the diameter of the tip.

Summarizing this section, we have demonstrated that the femtosecond NSOM technique can be used to study carrier dynamics in a single semiconductor quantum wire. Moreover, we have presented an experimental method for the mapping of energy fluctuations along a single quantum wire with high spatial resolution. With this method, quantitative information on the magnitude of quantum wire thickness fluctuations and on the length scale over which these fluctuations occur is obtained. These results demonstrate the potential of femtosecond NSOM for the characterization of nanostructured materials.

\section{Conclusions}

We have shown that femtosecond spectroscopy with spatial resolution beyond the diffraction limit can be performed if scanning near-field optical microscopy is combined with ultrafast pump-probe techniques. Today, the spatial and temporal resolution simultaneously achieved in near-field pump-probe measurements is of the order of $100 \mathrm{~nm}$ and $100 \mathrm{fs}$, respectively. Moreover, the results presented in this paper show that, with a well-engineered femtosecond NSOM instrument, a high sensitivity of $\simeq 10^{-4}$ can be obtained in near-field pump-probe measurements. For the future, further progress can be expected due to the recent development of high-throughput, small-aperture NSOM fibre probes [64, 65]. These NSOM probes should allow one to increase the spatial resolution without sacrificing sensitivity. Moreover, the temporal resolution can also be improved by building on the recent progress in femtosecond technology [66].

Already today, the combination of high spatial and temporal resolution and high sensitivity achieved in femtosecond NSOM is providing considerable insight into the spatiotemporal evolution of physical quantities. Typical femtosecond NSOM measurements can be placed into two categories: studies of single nanostructures and transport studies. As an example of the former, we have discussed femtosecond NSOM experiments on single semiconductor quantum wires. These measurements yield information on fluctuations of the quantization energy and the wire thickness along a single quantum wire. In general, the characterization of growth inhomogeneities in nanostructured materials is an important application of the femtosecond NSOM technique.

As examples of transport studies, we have presented experiments on laterally patterned semiconductor quantum wells and on a metal-semiconductor composite material. The latter consists of nano-scale tungsten discs embedded in GaAs. In the patterned quantum well samples, the details of carrier diffusion in square-like carrier density profiles have been observed. Carrier transport in the tungsten disc material has been found to involve both drift and diffusion of carriers. Although drift and diffusion of carriers have been studied 
by several groups $[23,25,26,28,31,32]$, ballistic transport phenomena have not yet been observed in femtosecond NSOM experiments. For semiconductors, ballistic transport in optical near-field experiments has been treated in detail theoretically [67-70]. Experimental studies of ballistic transport are a wide field for future femtosecond NSOM work.

\section{Acknowledgments}

The authors would like to thank B A Nechay and H Bielefeldt for setting up the ultrafast near-field scanning optical microscope and for their contributions to some of the experimental studies. We are also indebted to $\mathrm{F}$ MorierGenoud, A Schertel, A Hartmann, D Oberli, E Kapon and L-E Wernersson for providing us with various semiconductor samples. D Pohl and R Zenobi have given us access to their NSOM tip fabrication facilities. The femtosecond NSOM work was supported by the Swiss National Science Foundation under programme NFP 36.

\section{References}

[1] Shah J 1999 Ultrafast Spectroscopy of Semiconductors and Semiconductor Nanostructures (Berlin: Springer)

[2] Chergui M 1996 Femtochemistry (Singapore: World Scientific)

[3] Weisbuch C and Vinter B 1991 Quantum Semiconductor Structures: Fundamentals and Applications (San Diego: Academic)

[4] Kapon E 1997 Optical Spectroscopy of Low-Dimensional Semiconductors ed G Abstreiter et al (Amsterdam: Kluwer) p 99

[5] Bieler M, Hein G, Pierz K, Siegner U and Koch M 2000 Appl. Phys. Lett. 771002

[6] Pohl D W, Denk W and Lanz M 1984 Appl. Phys. Lett. 44651

[7] Betzig E and Trautman J K 1992 Science 257189

[8] Binnig G, Rohrer H, Gerber C and Weibel E 1982 Phys. Rev. Lett. 4957

[9] Binnig G, Quate C F and Gerber C 1986 Phys. Rev. Lett. 56930

[10] Hou A S, Ho F and Bloom D M 1992 Electron. Lett. 282302

[11] Nechay B A, Ho F, Hou A S and Bloom D M 1995 J. Vac. Sci. Technol. B 131369

[12] Nunes G and Freeman M R 1993 Science 2621029

[13] Weiss S, Ogletree D F, Botkin D, Salmeron M and Chemla D S 1993 Appl. Phys. Lett. 632567

[14] Takeuchi K and Kasahara Y 1993 Appl. Phys. Lett. 633548

[15] Botkin D, Glass J, Chemla D S, Ogletree D F, Salmeron M and Weiss S 1996 Appl. Phys. Lett. 691321

[16] Groeneveld R H W and van Kempen H 1996 Appl. Phys. Lett. 692294

[17] Keil U D, Jensen J R and Hvam J M 1997 Appl. Phys. Lett. 70 2625

[18] Steeves G M, Elezzabi A Y and Freeman M R 1998 Appl. Phys. Lett. 72504

[19] Donati G P, Rodriguez G and Taylor A J 2000 J. Opt. Soc. Am. B 171077

[20] Auston D H 1988 Ultrashort Laser Pulses and Applications ed W Kaiser (Heidelberg: Springer)

[21] Stark J B, Mohideen U, Betzig E and Slusher R E 1994 Ultrafast Phenomena IX ed P F Barbara et al (Heidelberg: Springer)

[22] Smith S, Orr B G, Kopelman R and Norris T 1995 Ultramicroscopy 57173

[23] Levy J, Nikitin V, Kikkawa J M, Cohen A, Samarth N, Garcia R and Awschalom D D 1996 Phys. Rev. Lett. 761948

[24] Vertikov A, Kuball M, Nurmikko A V and Maris H J 1996 Appl. Phys. Lett. 692465

[25] Smith S, Holme N C R, Orr B, Kopelman R and Norris T 1998 Ultramicroscopy $\mathbf{7 1} 213$
[26] Nechay B A, Siegner U, Morier-Genoud F, Schertel A and Keller U 1999 Appl. Phys. Lett. 7461

[27] Nechay B A, Siegner U, Achermann M, Bielefeldt H and Keller U 1999 Rev. Sci. Instrum. 702758

[28] Achermann M, Nechay B A, Morier-Genoud F, Schertel A, Siegner U and Keller U 1999 Phys. Rev. B 602101

[29] Guenther T, Emiliani V, Intonti F, Lienau C, Elsaesser T, Nötzel R and Ploog K H 1999 Appl. Phys. Lett. 753500

[30] Achermann M, Nechay B A, Siegner U, Hartmann A, Oberli D, Kapon E and Keller U 2000 Appl. Phys. Lett. 76 2695

[31] Achermann M, Siegner U, Wernersson L E and Keller U 2000 Appl. Phys. Lett. 773370

[32] Emiliani V, Guenther T, Lienau C, Nötzel R and Ploog K H 2000 Phys. Rev. B 61 R10 583

[33] Toda Y, Sugimoto T, Nishioka M and Arakawa Y 2000 Appl. Phys. Lett. 763887

[34] Kawashima H, Furuki M, Tatsuura S, Tian M, Sato Y, Pu L S and Tani T 2000 Appl. Phys. Lett. 771283

[35] Vollmer M, Giessen H, Stolz W, Rühle W W, Ghislain L and Elings V 1999 Appl. Phys. Lett. 741791

[36] Paesler M A and Moyer P J 1996 Near-Field Optics: Theory, Instrumentation, and Applications (New York: Wiley)

[37] Siegner U and Keller U 2000 Handbook of Optics vol III, ed M Bass et al (New York: McGraw-Hill)

[38] Lambelet P, Sayah A, Pfeffer M, Philipona C and Marquis-Weible F 1998 Appl. Optics 377289

[39] Stöckle R, Fokas C, Deckert V, Zenobi R, Sick B, Hecht B and Wild U P 1999 Appl. Phys. Lett. 75160

[40] Betzig E, Finn P L and Weiner J S 1992 Appl. Phys. Lett. 60 2484

[41] Karrai K and Grober R D 1995 Appl. Phys. Lett. 661842

[42] Duarte F J and Piper J A 1982 Opt. Commun. 43303

[43] Ziegler J F, Biersack J P and Littmark U 1989 The Stopping and Range of Ions in Solids vol 1 (New York: Pergamon)

[44] Lambsdorff M, Kuhl J, Rosenzweig J, Axmann A and Schneider J 1991 Appl. Phys. Lett. 581881

[45] Lederer M J, Luther-Davies B, Tan H H, Jagadish C, Haiml M, Siegner U and Keller U 1999 Appl. Phys. Lett. 741993

[46] Logan R A and Reinhart F K 1973 J. Appl. Phys. 444172

[47] Hecht B, Bielefeldt H, Inouye Y and Pohl D W 1997 J. Appl. Phys. 812492

[48] Knox W H, Fork R L, Downer M C, Miller D A B, Chemla D S, Shank C V, Gossard A C and Wiegmann W 1985 Phys. Rev. Lett. 541306

[49] Olsson A, Erskine D J, Zu X Y, Schremer A and Tang C L 1982 Appl. Phys. Lett. 41659

[50] Hillmer H, Forchel A and Tu C W 1992 Phys. Rev. B 451240

[51] Lam Y L and Singh J 1995 IEEE J. Quantum Electron. 31923

[52] Kim A M T, Hunsche S, Dekorsy T, Kurz H and Köhler K 1986 Appl. Phys. Lett. 682956

[53] Eichler H J, Guenter P and Pohl D W 1986 Laser-induced Dynamic Gratings (Berlin: Springer)

[54] Warren A C, Woodall J M, Freeouf J L, Grischkowsky D, Melloch M R and Otsuka N 1990 Appl. Phys. Lett. 571331

[55] Wernersson L-E, Carlsson N, Gustafson B, Litwin A and Samuelson L 1997 Appl. Phys. Lett. 712803

[56] Wernersson L-E, Litwin A, Samuelson L and Seifert W 1997 Japan. J. Appl. Phys. 36 L1628

[57] Whitaker J F 1993 Mater. Sci. Eng. B 2261

[58] Witt G L 1993 Mater. Sci. Eng. B 229

[59] Tommasi R, Langot P and Vallée F 1995 Appl. Phys. Lett. 66 1361

[60] Gustafsson A, Reinhardt F, Biasiol G and Kapon E 1995 Appl. Phys. Lett. 673673

[61] Abstreiter G, Aydinli A and Leburton J-P (eds) 1997 Optical Spectroscopy of Low-dimensional Semiconductors vol 344 (Amsterdam: Kluwer)

[62] Oberli D Y, Dupertuis M-A, Reinhardt F and Kapon E 1999 Phys. Rev. B 592910

[63] Fouquet J E and Burnham R D 1986 IEEE J. Quantum Electron. 221799 
[64] Eckert R, Freyland J M, Gersen H, Heinzelmann H, Schürmann G, Noell W, Staufer U and de Rooij N F 2000 Appl. Phys. Lett. 773695

[65] Kim B J, Flamma J W, ten Have E S, Garcia-Parajo M F, van Hulst N F and Brugger J 2001 J. Microsc. 20216

[66] Steinmeyer G, Sutter D H, Gallmann L, Matuschek N and Keller U 1999 Science 2861507
[67] Steininger F, Knorr A, Stroucken T, Thomas P and Koch S W 1996 Phys. Rev. Lett. 77550

[68] Steininger F, Knorr A, Thomas P and Koch S W 1997 Z. Phys. B $\mathbf{1 0 3} 45$

[69] Knorr A, Steininger F, Hanewinkel B, Kuckenburg S, Thomas P and Koch S W 1998 Phys. Status Solidi 206139

[70] Hanewinkel B, Knorr A, Thomas P and Koch S W 1999 Phys. Rev. B 608975 